\title{
Demanding an antibiotic for a cold is detrimental to your health, and mine
}

\section{Editorial}

This past winter, a reader visited the doctor for a stubborn cold. She was surprised when she was not prescribed an antibiotic. She asked why these drugs were not given for colds anymore? Caused by a host of viruses including the most likely culprit, Rhinovirus, Coronavirus, Respiratory Syncytial Virus, Parainfluenza, or any of the over 200 viruses, the common cold can be a miserable experience that few of us escape. If she was diagnosed with a cold, the physician knows that prescribing an antibiotic will do more harm than good. For one thing, it will have no impact on the virus, and may in fact destroy many commensal or "good bacteria" that are critical for boosting our immune systems. This is why physicians will generally not prescribe antibiotics to treat a viral infection. Aside from this, the bacteria in your digestive system, the microbiome, that allows you to process and extract nutrients from food, can be harmed or even destroyed by antibiotics, resulting in unpleasant conditions. Currently there are hundreds of antibiotics of various types and actions; however, on average a person will only encounter a handful of different types. Biologist Alexander Fleming returned to his office one September in 1928 to find he had left a petri dish of bacteria uncovered. The plate contained Staphylococcus bacteria, and Fleming noticed the bottom of the dish was covered with blue-green mold. He also noticed that a clear ring surrounded the mold, where the bacteria had been prevented from growing. The mold, Penicillin notatum, had somehow prevented the bacteria from attacking it. This original and entirely accidental discovery would set in motion the creation of one of the most used drugs in medical history.

Before the 1900's, physicians trying to treat patients with infections caused by bacteria (forms of pneumonia, gonorrhea, tuberculosis, rheumatic fever, urinary tract and kidney infections) lacked an effective treatment. By 1929 however, Fleming's discovery of penicillin changed the way medicine, and in particular, infectious diseases, were treated. Since that time, researchers have identified several antibiotic classes that combat bacteria and other bugs through some actions. These include Penicillins, Aminoglycosides,Cephalosporins, Macrolides, Fluoroquinolones, Sulfonamides, Tetracyclines, and Lincosamides. What you will be prescribed really depends on the suspected bug, and how your doctor chooses to treat it. The Penicillins are the oldest group of antibiotics, and their action is to inhibit the cell wall of the bacteria, blocking the synthesis of a molecule called peptidoglycan. Bacteria that have cell walls (some do not) contain a layer of peptidoglycan, and penicillin weakens the cell membrane causing cell death due to an inability to maintain the internal cellular environment.

Another common group of antibiotics are the Aminoglycosides include Vancomycin, Amikacin, Gentamicin, and Tobramycin. Similar to penicillin, these drugs also break down peptidoglycan, by in a slightly different way. The aminoglycosides are often used to treat acute infections caused by bacteria that either multiply very quickly or that have become resistant to other antibiotics.
Volume 7 Issue 3 - 2018

\author{
W Sumner Davis,' Karen Niemchick ${ }^{2}$ \\ 'Community Psychologist and Clinical Epidemiologist, Walden \\ University, USA \\ ${ }^{2}$ Grand Valley State University, USA
}

Correspondence: W Sumner Davis, Community Psychologist and Clinical Epidemiologist, Walden University, Oakland, ME 04963, USA, Tel +0I (207) 740-2028, Email dr.sumner.davis@gmail.com

Received: May 26, 2018 | Published: May 29, 2018

The cephalosporins are a group of antibiotics originally derived from the fungus Cephalosporum acremonium and are used to treat a wide range of infections including more severe diseases such as septicemia and bacterial meningitis. Newer, advanced generations of these drugs include Ceftaroline and Ceftolozane/tazobactam for treating highly resistant organisms like Methicillin-resistant Staphylococcus aureus (MRSA). Researchers working on ways to combat the evolution of bacteria have produced newer medicines that function in different ways. In 1952, scientists working with the bacterium Streptomyces erythreus were able to create the drug Erythromycin that inhibits bacterial protein synthesis of bacteria. Erythromycin derivative drugs are effective against Streptococci, Staphylococci, Clostridia, Corynebacteria, Listeria, Haemophilus sp., Moxicella, and Neisseria meningitidis. Clarithromycin and Azithromycin are useful in treating Mycoplasma pneumonia, and Helicobacter pylori, and are prescribed to treat obligate intracellular parasites (protists) including Toxoplasma gondiiand Cryptosporidia. While these drugs attack the ability of the pathogen to synthesize proteins to replicate, other groups of antibiotics disrupt the bacteria's DNA, creating a catastrophic failure in the replication process.

The fluoroquinolones area group of antibiotics that include Ciprofloxacin, Gemifloxacin, Levofloxacin, Moxifloxacin, Norfloxacin, and Ofloxacin. These broad-spectrum antibiotics can be used to treat a wide range of infections by selectively targeting the enzyme topoisomerase necessary in the winding and unwinding of DNA necessary for cellular replication. Introduced in the 1930s, the sulfonamides are bacteriostatic in that they inhibit the biosynthesis of folic acid essential for cell growth. Because these older drugs have been used for decades, many bacteria today have become resistant, but the drugs remain effective against many infections including urinary tract, as well as obligate parasite infections such as toxoplasmosis and some strains of malaria. Many Americans have been prescribed tetracycline antibiotics, including Doxycycline, which works by interfering with protein synthesis in the bacteria's cells which disrupts biosynthesis. Using a form of yeast called Streptomyces lincolnensis, scientists were able to isolate an enzyme that interferes with many bacterial RNA functions including disruption of the ability to replicate, and Lincomycin and Clindamycin have proven effective against several pathogenic species of Streptococcus, Staphylococcus, 
and Mycoplasma.

What is clear is that no antibiotic is effective against a virus, and expecting or demanding such a treatment for a virus is not only in effective but also detrimental to your health and is viewed as socially irresponsible. The over-prescribing of antibiotics in the last few decades, along with the significant use of antibiotics in factory farming, has created significant resistant strains of bacteria, a few of which there is currently no active treatment against. For example, the Centers for Disease Control and Prevention (CDC) has labeled drug resistant gonorrhea an urgent threat with 820,000 new infections annually in the U.S. and 246,000 of those showing resistance to at least one type of antibiotic (CDC, 2018). ${ }^{1}$ There are several things individuals can do. In the event your care provider prescribes an antibiotic, you should always take it exactly as directed, and finish the medication regardless if you feel better. Do not share any medication including antibiotics with others, and never save them for future use. Your doctor knows what infection you have, and has prescribed a specific type, strength, and number of antibiotics dispensed by the pharmacist to treat it. Should you have further questions on antibiotics, antibiotic resistance, or antibiotic use, consult your physician or care provider, or by consulting reputable information sources such as MedLine Plus, the World Health Organization, or the Centers for Disease Control and Prevention.

\section{Acknowledgements}

None.

\section{Conflict of interest}

The author declares that there is no conflict of interest.

\section{References}

1. Centers for Disease Control and Prevention. Antibiotic/Antimicrobial Resistance. (2018). 